\title{
Mediated Communication and Customer Service Experiences Psychological and Demographic Predictors of User Evaluations in the United States
}

\author{
Kate K. Mays ${ }^{1 *}$, James E. Katz¹, Jacob Groshek² \\ 1 Division of Emerging Media Studies, College of Communication, Boston University, 640 Commonwealth Avenue, Boston, \\ Massachusetts 02215, United States of America \\ 2 A.Q. Miller School of Journalism and Mass Communications, Kansas State University, 105 Kedzie Hall, Manhattan, Kansas 66506, \\ United States of America \\ * Corresponding author, e-mail: kkmays@bu.edu
}

Received: 21 July 2020, Accepted: 21 October 2020, Published online: 20 December 2021

\begin{abstract}
People around the world who seek to interact with large organisations increasingly find they must do so via mediated and automated communication. Organisations often deploy both mediated and automated platforms, such as instant messaging and interactive voice response systems (IVRs), for efficiency and cost-savings. Customer and client responses to these systems range from delight to frustration. To better understand the factors affecting people's satisfaction with these systems, we conducted a representative U.S. national survey $(N=1321)$. We found that people overwhelmingly like and trust in-person customer service compared to mediated and automated modalities. As to demographic attitude predictors, age was important (older respondents liked mediated systems less), but income and education were not strong attitude predictors. For personality variables, innovativeness was positively associated with mediated system satisfaction. However, communication apprehensiveness, which we expected to be related to satisfaction, was not. We conclude by discussing implications for the burgeoning field of human-machine communication, as well as social policy, equity, and the pullulating digital services divide.
\end{abstract}

\section{Keywords}

automation, interactive voice response systems, IVRs, customer service, social media, chatbots

\section{Introduction}

Over two decades ago, Katz et al. (1997) investigated the phenomenon of electronic voice messaging systems, then referred to as VRUs (voice response units). It was a technology emerging predominantly in corporations' customer service arms aiming to cut costs and boost efficiency. Despite VRUs' promise, customers themselves were generally unenthusiastic about the new technology and found it unproductive and frustrating.

In the time since, automated services like Interactive Voice Response systems (IVRs) and chatbots have continued to be hailed as cost- and time-savers for companies' customer service operations (Buesing et al., 2019). For rote inquiries that can be more easily standardised, IVRs have been used to supplement or even replace paid employees in receptionist or customer service roles. IVRs can re-direct calls to the appropriate departments, schedule appointments, refill prescriptions, provide account information, among many other information, coordination and communication-oriented tasks.

If one googles "IVR customer service uses", a plethora of results pop up with optimistic prognostications of improved customer satisfaction and more efficiency for both the customer and the company from an error-free, streamlined process that can handle a high volume of calls, ultimately increasing productivity and profits for the company.

However, the customer service provider Startek found in 2017 that $85 \%$ of respondents to its survey preferred interacting with humans over automated agents like IVRs or chatbot/AI platforms, with exceptions for basic inquiries like checking one's account balance. These preferences seemed to be driven by people's desire for empathy in a customer service interaction (STARTEK, 2017): with 
human empathy on the other side, there's a chance that rigid process may by eased, context considered, and therefore exceptions made for a person's particular situation.

It has been argued that people's frustration with IVRs derives from the lack of power they have in these interactions; in effect they become supplicants required to interact with a non-human, unintelligent entity. Thus IVRs seem to act primarily as "gatekeepers to information" (Walsh et al., 2018), requiring the customer to perform a number of steps and fulfil certain requests before they can get the help they initially sought. As such, customers are "faced with a dilemma; they need help from the institutions but dislike IVR interactions, yet despite their dislike, they are routinely forced to engage with the system to achieve their desired ends" (Walsh et al., 2018). In their qualitative exploration of people's experiences with and perceptions of IVR, Walsh and colleagues found that their participants had a range of strategies to "work with" or, with more experience, "circumvent" the IVR technology (Walsh et al., 2018).

Their participants' acquiescence and subsequent circumvention strategies to effectively navigate IVRs suggests what seems clear from industry trends: IVR and other AI-enabled automated systems are not going away. Rather, the ways for people to access information and resources are proliferating, and for some this is revolutionising their lives. Accessing information now with technology is not simply learning the weather forecast but actual problem-solving to navigate through daily life. Further, technology is vastly different from the simpler search interfaces and electronic messaging systems that predated our automated age. These information systems no longer only convey messages between people as mediators, but in some instances act as communication partners themselves, which may require a reconceptualisation of communication technologies beyond the computer-mediated communication (CMC) paradigm (Gunkel, 2012). This new age of Artificially Intelligent (AI) communication devices compels us to think about human communication beyond face-to-face and mediated modalities, and think about human-machine communication (Guzman and Lewis, 2020; Spence, 2019).

The fact is that in the coming decades, vast amounts of our lives will be run algorithmically, with humans' voices serving as a major machine interface. Increasingly sophisticated computers and interfaces will address an array of human needs through automatic services. However, experience shows that, even after years of iteration, these services are far from flawless; seemingly people will continue to need customised interventions to address specific situations and problems. Too, research shows that people are (understandably) hesitant to turn over important decisions to "faceless" computers.

While these trends concerning IVRs are occurring worldwide, in this study we look at the United States as a bellwether to see how people are doing now by comparing how people perceive different modalities for accessing information to solve problems in support of their personal needs. Through a nationally representative survey, we compared and contrasted perceptions of these services' utility as modelled through individual-level variables such as communication apprehension, innovativeness, and experience with them. Given the encroaching ubiquity of these services in our lives, the implications of our findings are relevant to systems designers, social scientists, and people concerned about social policy and equity.

\section{Literature review}

There are existing models that explain part of people's approach toward new technologies in terms of acceptance, such as through models like the "Technology Acceptance Model" (TAM) and "Technology Readiness" (TR). These models are helpful in parsing how people's particular perceptions of a specific technology (e.g., how easy it is to use and their intentions to use it) inform their actual use of the technology, and the amount of variance they are able to explain demonstrates their utility in predicting whether or not people will use a technology based on how they perceive it. However, for this study we are interested in understanding individual, trait-based correlates of attitudes about different modalities of customer service, one of which is in-person and the other two are technology based.

\subsection{Media richness}

Modality is important to consider because communication channels have different affordances that influence perception and use. Media richness theory asserts that the more cues a communication channel has, the more it is able to reduce uncertainty and ambiguity (Daft and Lengel, 1986). It was originally proposed in order to explain the effects of different types of media on task performance and was subsequently applied to new media that emerged in the 1990s and beyond, as the range of possible communication channels expanded with online and digital platforms. Research focused on communication channels' different attributes and the extent to which they could transmit 
types of information. Media richness theory's core proposition was that the more information and content dimensions a medium provided - the "richer" a medium was the more that medium would be perceived as satisfying and effective (Caplan et al., 2014). To put it another way, different channels provide varying amounts of social presence, a critical lubricant for interpersonal communication (Campbell, 2006). Face-to-face interaction would be considered the "richest" way to communicate because it can transmit verbal and nonverbal cues in numerous ways (e.g. haptically, visually, aurally), which mitigates misunderstandings (Campbell, 2006; Daft and Lengel, 1983). Following face-to-face, telephone (audio) is the next highest in communication richness, followed by electronic and written forms of communication (Daft and Lengel, 1983; Vickery et al., 2004).

Customer service now plays out across a spectrum of communication channels. There is still face-to-face or "in-person" customer service, whereby someone interacts with another person, and then there are varying degrees of mediated customer service: over e-mail, social media platforms, and instant messaging chats. In these exchanges, another person is at the other end of the interaction, but the communication is less rich because of the limited visual/ audio cues and, in some cases, less instantaneous feedback. IVRs are distinct from computer-mediated communication (CMC) in that they are not computer systems passing information between two people, but rather are secondary communication entities in exchanges. In some cases, IVRs are a communicative gatekeeper prior to an eventual person-to-person exchange, such as when IVRs serve to direct inquiries to appropriate departments (Miller et al., 2013).

Because it uses voice to relay information, IVRs traditionally would be considered a "richer" medium than CMC, which only relies on text. Indeed, when comparing digital customer service channels (text, audio-only, and video), satisfaction corresponded with the digital channel's level of richness (Gimpel et al., 2016). Therefore, one might expect that automated customer service channels like IVR would be preferred over mediated customer service. However, media richness also takes into account how personal or impersonal the communication source is (Daft and Lengel, 1983). To our knowledge, this aspect of media richness has not been explored with regards to automated technology that can simulate human-ness but is clearly automated.

Indeed, scholars have argued that traditional communication theories predicated on human-human interaction may not directly apply to human-machine communication
(HMC) (Gunkel, 2012; Guzman and Lewis, 2020). Media richness theory was developed at the early stages of the online communication revolution. Since then, alternative theories have emerged to explain how and why people seemed to be using online interfaces to form relationships and interact socially (Halpern and Katz, 2013). For example, social information processing (SIP) theory contends that, given enough time, people can communicate just as intimately and effectively through online means because people will adapt to the medium (Walther, 1992; 1994).

With the addition of automated interfaces, though, it is not clear, whether the same tenets of media richness apply when people are talking with machines, rather than through them to a human on the other side (Guzman and Lewis, 2020). Increasingly, people are interacting with AI technologies - such as IVRs in a service setting or digital voice assistants like Alexa in a domestic setting through the course of their daily lives (Guzman, 2018). A meta-analysis has shown that people are more influenced by human-controlled virtual agents than they are by computationally controlled agents, suggesting a preference for a "human touch" in communication technology (Fox et al., 2015).

Importantly, people's baseline expectations for robot interactions, as an example of another AI entity, were found to be lower in terms of anticipated liking, uncertainty, and social presence (Spence et al., 2014). These findings were consistent in a follow-up study that also showed participants an image of a mechanistic robot interactant (Edwards et al., 2016). However, a third study showed that when the robot appeared more human, uncertainty decreased and expectations for social presence increased (Edwards et al., 2019). Putting these results together, the researchers have proposed an "anthropocentric expectancy bias" for communication that might explain why people are much less comfortable with the abstract idea or mechanistic image of a robot, as compared with a more human-looking robot interactant.

Further, it has been a few decades since these theories were first formulated, and in that time, people have continued to use and innovate online and other digital platforms; some now have had lifelong acquaintanceship with these technologies. Presumably there has been a great deal of acclimation and learning taking place, wherein these technologies are less foreign as people have mastered them. Context may matter, also. For example, in existing business-to-business relationships, electronic media could be modelled as a similarly rich media to telephone 
and face-to-face communication (Vickery et al., 2004) and within organisations, different tasks are more conducive for richer or leaner media depending on the nature of the task (Rice, 1992). We therefore ask the following research question about how attitudes towards customer services may differ.

RQ1: Are there differences in attitudes toward customer service modalities that vary in their media richness?

\subsection{Individual traits}

Media richness primarily focuses on how the channel's attributes affect perception and use. It is also important to consider the ways in which individual characteristics may influence perception and usage. It may be that the richness of a medium is not fixed with a uniform effect, but rather that people may differ in their perceptions of a medium's richness - and thus their satisfaction with the medium - based on personal traits and past experiences (Pieterson et al., 2008). Previous research has shown that experience with certain technologies improves people's attitudes (Katz et al., 1997; Rosen et al., 1993) and reduces their anxiety about using them (Orr et al., 2001).

Communication apprehension has been linked to more computer anxiety generally (Brown et al., 2004). When comparing videoconferencing and face-to-face meetings, people higher in communication apprehension liked the mediated meeting format less, which was explained potentially by their heightened self-awareness and anxiety about losing face in front of their peers (Campbell, 2006). However, these apprehensive individuals regarded the technology more positively after regular use. In newer media contexts, individuals with higher communication apprehension in a CMC context were less motivated to use Facebook for interpersonal communication, which was related to less use of Facebook's interactive features (Hunt et al., 2012).

Innovativeness was originally conceptualised as a personality trait that indicated one's willingness to change (Hurt et al., 1977). It has since been identified as a persistent trait that corresponds with openness to and adoption of new technologies (Yi et al., 2006). Rogers (2003) included individual innovativeness in his model for diffusion of innovations and created an "innovator" category of adopters who outpace the rest in terms of openness to new things. The research on how individual innovativeness relates to technological acceptance and adoption is mixed, however. Individual innovativeness has been found to be a significant predictor of intended technological use (Yi et al., 2006), and more innovative teachers, for example, were more likely to use computers in their classroom (Van Braak, 2001). In a different study, though, domain-specific innovativeness was more likely to predict product adoption as compared to innate innovativeness, which appeared to have no effect (Chao et al., 2012). Looking at more sustained use, beyond initial adoption, innovativeness may not have as much of an influence (Doong et al., 2010). Given the mixed findings on how personality influences technology acceptance and adoption, we pose the following research question.

RQ2: To what extent do people's a) experience, b) communication apprehension, and c) innovativeness influence their perceptions of different customer service modalities?

\section{Method}

In early fall 2015 we conducted a nationally representative survey (along four demographic categories - age, gender, education, and ethnicity, $N=1321$ ) of American respondents that probed their recent customer service experiences and their general opinions about different customer service modalities (e.g. in-person, mediated channels like e-mail and social media, as well as and automated services like IVR and chatbots for customer service). To the maximum feasible extent, in all regards we attended to the standard best practices for surveys set forth by the American Association for Public Opinion Research (AAPOR). The survey was administered as an online questionnaire through the professional survey company Qualtrics, which recruits American adults for compensation to participate in surveys. Our sample had an average age of 29.36 $(S D=16.33)$ and was $51 \%$ female. Respondents were predominantly white/Caucasian (68\%); 66.5\% had at least some college through a 4-year degree; and $87 \%$ had an annual household income of less than $\$ 100,000$.

\subsection{Attitudes about customer service across modalities}

We measured customer service attitudes with an index that asked for general opinions of different types of customer service (in-person; telephone with a person; e-mail; social media; instant messaging; IVR; virtual assistant; live chat) on a 4-point Likert-type scale ("miserable", "unsatisfactory", "satisfactory", "excellent", system-missing = "never used / don't know what it is"), as well as level of trust in each modality, also on a 4-point Likert-type scale ("none", "a little", "some", "a lot", system-missing = "never used / don't know what it is"). We combined these items to create scales for three different modalities by averaging together 
the opinion and trust items. Here, it is important to point out that the usage reported for each modality is specific to uses related to customer service, and not media use more generally (such as social media or virtual assistants that may have been used more frequently in other contexts).

\subsubsection{In-person customer service}

This measure included both a live person over the phone and an in-person interaction (4 items, $\alpha=0.815$ ). The highest number of respondents $(\mathrm{n}=1196)$ provided their assessment of this modality; only about $8 \%$ of the sample responded that they didn't know or had never used it $(M=3.42, S D=0.58)$.

\subsubsection{Mediated customer service}

This measure combined attitudes about customer service via e-mail, instant chat, or social media (6 items, $\alpha=0.829$ ). This modality appeared to be the least well known, as almost $39 \%$ of respondents $(n=695)$ had never used or didn't know about social media customer service $(M=2.69, S D=0.65)$ Opinion and trust - mediated platforms (email, chat, social media).

\subsubsection{Automated customer service}

This measure encompassed IVR and virtual assistant technologies, as well as instant chat with an automated computer agent like a chatbot ( 6 items, $\alpha=0.884$ ). About $37 \%$ of respondents $(n=709)$ had never used or didn't know about virtual assistant customer service $(M=2.46, S D=0.79)$.

\subsection{Predictors of attitudes}

Experience: Past research (Katz et al., 1997; Pieterson et al., 2008) has shown that people's past experiences with customer service technology can inform their general perceptions and attitudes about the technology. Therefore, we included three measures that asked respondents about:

1. how recent their last customer service experience was (7-point scale, Never - within the last week);

2. how they would rate that experience (4-point scale, Miserable - Excellent); and

3. how likely they would be to continue using the company and recommend it to a friend ( 3 items, 5-point scale "very unlikely" to "very likely", $\alpha=0.852$ ).

It is important to note that $88.4 \%$ of respondents $(n=1168)$ reported their most recent customer service experience as having been either a mediated or automated interaction.
Individual traits: The communication apprehension index was adapted from McCroskey's (2005) "Personal Report of Communication Apprehension" scale. The fivepoint ("strongly disagree" to "strongly agree"), four-item scale $(\alpha=0.800)$ included statements such as: "Generally, I am comfortable while participating in group discussions.", "Communicating at meetings usually makes me uncomfortable" (reverse coded). Items were coded such that a higher score on the communication apprehension index indicated less apprehension, i.e. more comfort when communicating $(M=3.44, S D=0.96)$.

The measure for innovativeness was adapted from Hurt et al. (1977). It is a five-point ("strongly disagree" to "strongly agree") five-item Likert-type scale $(\alpha=0.729)$ that included statements such as: "I enjoy trying new ideas" and "I often find myself sceptical of new ideas" (reversecoded). Items were coded such that a higher score indicated higher innovativeness $(M=3.69, S D=0.65)$.

Age, gender, education, and income were also included as controls in the models, given prior research showing to varying degrees that these characteristics influence attitudes towards technology (Katz et al., 1997).

\subsection{Analysis}

All analyses were conducted using IBM SPSS Statistics. We first ran frequencies to get a picture of the data and relationships between variables. Then we constructed hierarchical linear regression models to evaluate predictors of respondents' attitudes towards the three different customer service modalities.

\section{Results}

\subsection{Attitudes towards customer service modalities}

Respondents' general opinions and levels of trust in the different modalities for customer service are reported in Figs. 1 and 2. As can be seen, in-person customer service is still vastly preferred over the mediated and automated options. Over $80 \%$ of respondents were at least satisfied with in-person interactions; in comparison, roughly $60 \%$ of respondents were at least satisfied with e-mail or instant chat interactions, and only $30-40 \%$ of respondents were at least satisfied with automated customer service. These proportions are similar for those who have at least "some" level of trust in the various customer service modalities.

As these descriptions might suggest, there were significant differences between each measure of customer service perception, with the biggest difference in means between in-person customer service and automated 


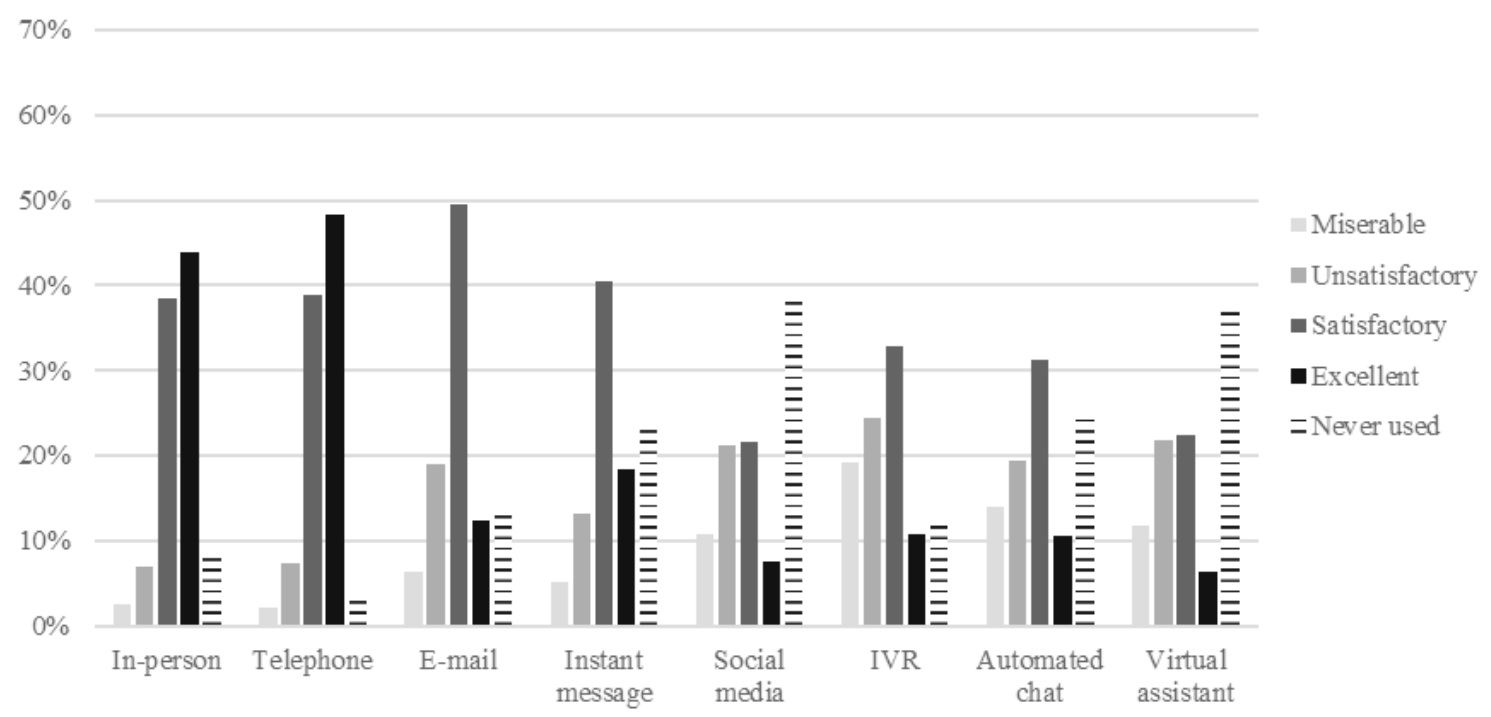

Fig. 1 General opinion of customer service modalities

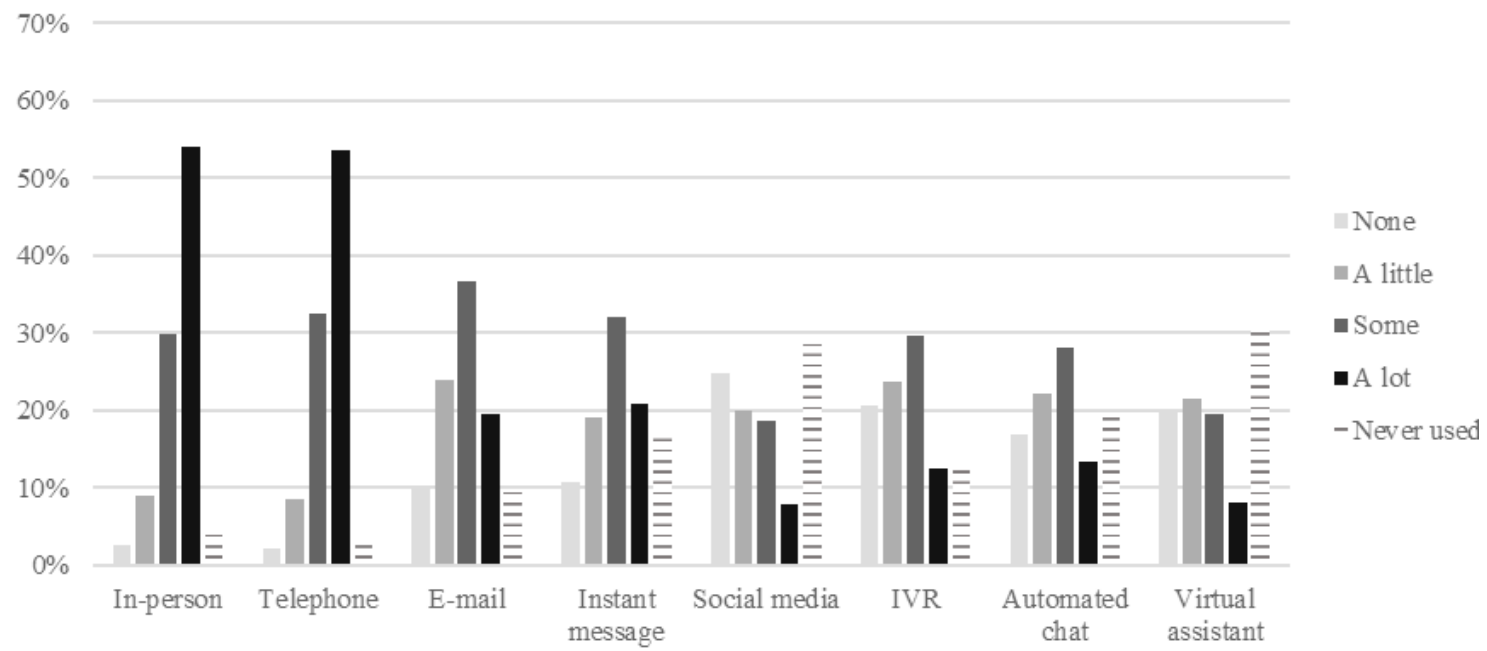

Fig. 2 Trust in customer service modalities

customer service (paired $t(696)=29.13, p<0.001$ ). There was the smallest difference between mediated and automated customer service (paired $t(578)=7.62, p<0.001$ ). Indeed, mediated and automated customer service attitudes were relatively strongly correlated with one another $\left(r_{s}=0.69, p<0.001\right)$.

Interestingly, though in-person customer service is highly preferred, only $5 \%$ of respondents $(n=65)$ had an in-person interaction as their last customer service experience. In this respect, automated services seem to be winning out: $58 \%$ of respondents $(n=759)$ had some kind of automated interaction as their last customer service experience, while $31 \%$ of respondents $(n=409)$ had some kind of mediated interaction as their last customer service experience.

\subsection{Modelling customer service attitudes across modalities}

To understand the contribution of individual traits to customer service attitudes across modalities, we constructed a series of hierarchical OLS regressions for each. To reiterate, the dependent variables for each of these modalities (in-person, mediated, and automated) customer service experiences were summarised indexed based on 4-point Likert scales as described in Subsection 3.1 previously.

Each of these models contained three blocks:

1. demographic characteristics (age, gender, education, income);

2. individual traits (communication apprehension and innovativeness); and

3. recent customer service experience. 
With the exception of the demographics block in the in-person customer service model, all blocks in the three models were significant at $p<0.001$.

As can be seen in Table 1, the differences were most distinct between in-person customer service and the other two modalities. The predictors explained the most variance for mediated customer service $(23.7 \%)$, followed closely by automated customer service (19.0\%); only $11 \%$ of the variance in in-person customer service was explained by the included variables.

For attitudes toward in-person customer service, innovativeness $(\beta=0.163, p<0.001)$ and satisfaction with one's most recent experience with customer service $(\beta=0.196$, $p<0.001$ ) were the strongest positive predictors. Age was also significant - the older someone was the more positively disposed they were to in-person customer service ( $\beta=0.082, p<0.001)$. This pattern is reversed for mediated and automated customer service: age was negatively correlated with attitudes toward mediated $(\beta=-0.226$, $p<0.001)$ and automated $(\beta=-0.199, p<0.001)$ modalities.

Personality traits contributed the least amount of explanation towards attitudes of mediated and automated customer service, and of these, innovativeness was the only significant predictor of positive attitudes towards mediated $(\beta=0.133, p<0.001)$ and automated $(\beta=0.109, p<0.001)$ modalities. Satisfaction with and loyalty to a company

Table 1 Predictors of attitudes towards different customer service modalities

\begin{tabular}{|c|c|c|c|}
\hline & $\begin{array}{c}\text { In-person } \\
\beta\end{array}$ & $\begin{array}{l}\text { Mediated } \\
\qquad B\end{array}$ & $\begin{array}{c}\text { Automated } \\
\qquad \beta\end{array}$ \\
\hline Age & $0.082 * * *$ & $-0.226^{* * *}$ & $-0.199 * * *$ \\
\hline $\begin{array}{l}\text { Gender } \\
(1=\text { male, } 2=\text { female })\end{array}$ & 0.021 & -0.059 & $-0.109 * * *$ \\
\hline Income & -0.004 & -0.012 & -0.036 \\
\hline Education & $-0.069^{*}$ & $0.066)$ & 0.052 \\
\hline$R^{2}$ change & $0.4 \%$ & $7.8 \% * * *$ & $5.6 \% * * *$ \\
\hline $\begin{array}{l}\text { Communication } \\
\text { apprehension }\end{array}$ & $0.062^{\dagger}$ & -0.031 & -0.008 \\
\hline Innovativeness & $0.163 * * *$ & $0.133^{* * *}$ & $0.109^{* *}$ \\
\hline$R^{2}$ change & $5.0 \% * * *$ & $3.1 \% * * *$ & $2.0 \% * * *$ \\
\hline $\begin{array}{l}\text { Recent customer } \\
\text { service interaction }\end{array}$ & 0.029 & 0.052 & -0.037 \\
\hline $\begin{array}{l}\text { Recent customer } \\
\text { service satisfaction }\end{array}$ & $0.196 * * *$ & $0.209 * * *$ & $0.193 * * *$ \\
\hline $\begin{array}{l}\text { Recent customer } \\
\text { service loyalty }\end{array}$ & 0.067 & $0.188 * * *$ & $0.188 * * *$ \\
\hline$R^{2}$ change & $6.2 \% * * *$ & $13.8 \% * * *$ & $12.5 \% * * *$ \\
\hline Total adjusted $R^{2}$ & $11.0 \%$ & $23.7 \%$ & $19.0 \%$ \\
\hline
\end{tabular}

Note: $\beta=$ standardised regression coefficient; ${ }^{\dagger} p<0.10,{ }^{*} p<0.05$, $* * p<0.01, * * * p<0.001$ based on the most recent customer service interaction had a nearly identical influence and positively predicted attitudes, explaining $13.8 \%$ and $12.5 \%$ of the variance in mediated and automated customer service, respectively.

\section{Discussion}

Information today is communicated through myriad channels. As ever more AI-enabled technology has emerged, communication occurs not only with other humans through technology, but also with the technology itself. Access to and competence with technology remain important considerations for social equity and progress. To that end, this study examined attitudes towards different technologies in a customer service context. As companies attempt to cut costs and improve performance, they have turned to automated systems like IVR to offload customer service tasks. Through a U.S. national survey, we compared attitudes about different customer service modalities that ranged in their levels of "richness": in-person (face-to-face or over the phone), mediated (e-mail, social media, instant messaging), and automated (IVR, virtual assistant, chatbot).

We found that people still overwhelmingly like and trust in-person customer service over mediated and automated modalities. This finding aligns with the main proposition of media richness, which is that the number of cues a channel has corresponds with its ability to convey information, and cue-heavy channels are preferred over those lighter on cues. One limitation to our approach is that we did not ask for perceptions of richness from respondents; rather, we categorised the channels based on their similarity of features.

Interestingly, mediated customer service was preferred slightly more than automated services such as IVR, even though mediated channels technically have fewer cues than IVR (e.g., text only). It seems, therefore, that the automated aspect of IVR-type technology is a crucial component of people's attitudes. This follows what a qualitative investigation of attitudes on IVR found in terms of people's frustration with automated customer service and their belief that interacting with a human was bound to result in more favourable outcomes.

The AI component's apparent importance suggests that more is needed theoretically beyond media richness to explain these findings. A channel's additional cues may help reduce uncertainty and ambiguity, as proposed by media richness, but if the channel's source of information like an automated agent is perceived as not credible, then uncertainty may increase regardless of the amount of information 
conveyed. Thus, the cognitive processing that underlies the information cues may be critical (Sundar, 2007). Information cues in this context, therefore, can be conceptualised as technological affordances that provide the user with more than just information, but also feelings of agency and control over their digital interactions.

In particular, Sundar's (2007) "machine heuristic" shows how information source cues influence perceptions and behaviour. The machine heuristic proposes that when people think they are interacting with a machine rather than a human, they attribute machine-like qualities (e.g., objectivity, neutrality, systematic) to the interaction and their outcome expectations. The machine heuristic has positively influenced people's personal information disclosure online - they are more likely to share private information with a machine rather than a human agent, particularly if they believe more in the machine heuristic (Sundar and Kim, 2019). This has been theorised particularly in the context of news credibility, where ideological bias is an ongoing concern. Early research demonstrated that news selected by a machine was perceived more credibly than news chosen by a human editor (Sundar and Nass, 2001). More recently, however, findings about machine intervention in news production are mixed: some have found that news written or selected by a machine and a human is perceived equivalently (Clerwall, 2014), while others have found that news written by a machine, compared to a human, was seen as less credible, thus refuting the machine heuristic explanation in favour of expectancy violations (Waddell, 2018; 2019). As such, context appears to be important: depending on people's goals, different qualities for an interactant may be desired.

In customer service contexts, for example, people may want the presence or possibility of bias, or at least individualisation. Someone accessing customer service is more likely to already have a special or edge case for which they want or expect customised treatment. Optimistically, they may hope that any bias will lean in their favour: a person is better able than a machine to both know how to, and choose to, skirt around the rules to advocate for the customer (Walsh et al., 2018). Person-person persuasion may seem more feasible that person-machine persuasion, whereby machines are perceived as rigid and immovable.

To that end, in their qualitative work Walsh et al. (2018) identified feelings of agency as a key component of people's resistance to automated customer service platforms. Participants felt that they were less adept with navigating an automated response than a human one, and some discussed how, over time, they had learned how to develop new navigational shortcuts. Without agency, one loses the ability the control the surrounding environment and outcomes. Feeling in control is an important component of people's experiences in digital environments; affordances like interactivity and navigability ultimately afford control to users (Kalyanaraman and Wojdynski, 2015; Sundar, 2007).

We therefore suggest that a "human heuristic" may be a corollary to the "machine heuristic" with regards to AI-driven technology in certain contexts. HMC scholars have identified an "anthropocentric expectancy bias" that is violated when people have to interact with a machine instead of a human (Edwards et al., 2016; 2019; Spence et al., 2014). This bias could feed assumptions people make when engaging with automated agents in contexts when human qualities (flexibility, nuance, bias) are desired. Further, avatars (human-controlled virtual agents) have been found to be more influential than computer-run agents, particularly in competitive and cooperative tasks (Fox et al., 2015), supporting the notion that context for human-machine communication is important.

Researchers have suggested that AI should be treated as a unique kind of communicator that does not parallel human communication (Guzman and Lewis, 2020). Indeed, some have argued that communication research moving further into the $21^{\text {st }}$ century must adapt or develop new theoretical paradigms to understand technology not only as a neutral medium that conveys information, but that serves as an "information source or receiver" (Gunkel, 2012). Specifically, AI-driven technology challenges the dominant $\mathrm{CMC}$ conceptualisation of technology primarily as a tool to facilitate human-to-human communication. It may be this instrumentalist perception of technology in particular that frustrates people in the especially transactional environment of customer service.

Further, the findings suggest, as HMC scholars have argued, that it may not be appropriate to directly compare automated machine communication with human-human communication, with the assumption that human communication is always the "gold standard" (Spence, 2019). While the results in this study bear that out participants vastly preferred human-human communication, either in person or mediated, over automated communication, this comparison may not be the most productive path for enhanced understanding HMC. We would argue that although there is no need to disregard CMC theories, researchers should be careful when building on them to not just consider automated technology as yet another 
iteration of a mediated modality (Gunkel, 2012). The findings provide support for the argument that HMC should be conceptualised alongside but apart from the existing CMC paradigm, rather than only from within it.

It also follows that recent customer service experience was most strongly related to positive attitudes, aligning with research that has shown that positive prior experiences with technology results in more positive attitudes towards the technology (Katz et al., 1997). Over 90\% of our sample had most recently accessed customer service through mediated and automated means. While it would have been ideal to split out these samples based on these experiences and make comparisons, we kept them grouped together to retain our models' statistical power.

In terms of individual characteristics, we found that innovativeness was positively related to attitudes across all three customer service modalities. And while one can only speculate as to why this characteristic persisted not just with mediated and automated modalities but also with in-person experiences, it could simply be that those individuals were more willing to work to find acceptable solutions, regardless the interface. Alternatively, higher education levels were negatively related to in-person customer service but neither of the other modalities, which may potentially imply a certain power distance between more educated customers and service representatives. To speculate, it might be that non-human modalities are seen by customers as class-neutral or emotionally neutral, thus not invoking threats to the customer's self-image or feelings of deference. If this finding is borne out, it presents an area teeming with implications.

Communication apprehension had no major effect, though it was weakly correlated with in-person customer service (e.g. those who were less apprehensive held more positive attitudes towards in-person customer service). Not surprisingly, age was negatively correlated with attitudes towards both kinds of customer service technologies. Future studies might look further into what other personal traits contribute to perceptions of automated technologies, which could then inform better individual customisation of such technologies.

Of course, our study was also limited by the inherent shortcomings of survey methodologies and attitude measurement, the defects of which are well rehearsed in the literature. Additionally, we recruited respondents through a professional survey company, and it was administered online. While the sample's demographic distribution matched that of the United States, there may be other characteristics that mitigate the ultimate representativeness of the sample - and thus generalisability of the survey's findings. As to the time dimension, this survey was conducted in late 2015. Some technologies have been subsequently modified, and people may be more accustomed to interacting with automated customer service today after a few more years interacting with the technology. That said, the mediated forms of customer service that this study examined - e-mail, instant messaging, and social media - have been around longer and it was still preferred less than in-person customer service. Therefore, we believe the findings of the correlates should still be of value. One reason for this belief is that general data concerning these relationships have been largely unavailable and, in the case of our findings, are presented here for the first time. Secondly, many of the relationships we detected are likely to be quite stable, similar to the cases of other mediated technologies. Finally, the information presented here has broader applicability given that mediated communication technology interfaces are becoming more common worldwide, especially in the developing world, and therefore is worthy of dissemination.

As automated technology and artificial intelligence expands further into our lives, much in the same way that digital technology has in the last quarter century, it will be important to understand how this modality enables and hinders people's individual effectiveness and satisfaction, as well as their socio-economic prospects. A digital information divide may widen further to encompass a digital services divide. This study constitutes a step towards understanding factors that contribute to the prevention or amelioration of problems related to service access as technologies of personal power proliferate.

Theory in this area is frequently built without reference to any systematic data and instead relies on examples. Having both independent empirical support from a statistic-ally representative population is a valued addition in the conceptual development in a new area such as HMC, as set forth by others (Gunkel, 2012; Guzman and Lewis, 2020; Spence, 2019). While we speculate here that the discrepancy in perceptions of these technologies is based on heuristics - the assumption that lack of humanness in customer service will be rigid and frustrating - the differences may simply be based on lived experiences. Future research should experimentally examine this "human heuristic" with automated agents to determine whether it operates similarly as the machine heuristic in digital spaces. Although the results have yet to be independently 
confirmed, they suggest an exciting data-supported development that may prove to be fruitful. Isolating the mechanisms by which automated technology is more or less liked and taken up will have important implications; this is particularly true with the dimension of power dynamics and other traditional sociological concerns like class, ethnicity, and gender.

\section{References}

American Association for Public Opinion Research (AAPOR) "Best Practices for Survey Research", [online] Available at: https://www.aapor.org/Standards-Ethics/Best-Practices.aspx [Accessed: 20 July 2020]

Brown, S. A., Fuller, R. M., Vician, C. (2004) "Who's Afraid of the Virtual World? Anxiety and Computer-Mediated Communication", Journal of the Association for Information Systems, 5(2), pp. 79-107. https://doi.org/10.17705/1jais.00046

Buesing, E., Gupta, V., Kleinstein, B., Mukhopadhyay, S. (2019) "Getting the best customer service from your IVR: Fresh eyes on an old problem", McKinsey \& Company [online] Available at: https://www.mckinsey.com/business-functions/operations/ourinsights/getting-the-best-customer-service-from-your-ivr-fresheyes-on-an-old-problem [Accessed: 20 October 2020]

Campbell, J. (2006) "Media Richness, Communication Apprehension and Participation in Group Videoconferencing", Journal of Information, Information Technology and Organizations, 1, pp. 87-96.

https://doi.org/10.28945/149

Caplan, S. E., Perse, E. M., Gennaria, J. E. (2014) "Computer-Mediated Technology and Social Interaction", In: Lin, C. A., Atkin, D. J. (eds.) Communication Technology and Social Change: Theory and Implications, Routledge, New York, USA, pp. 53-72.

Chao, C. W., Reid, M., Mavondo, F. T. (2012) "Consumer Innovativeness Influence on Really New Product Adoption", Australasian Marketing Journal, 20(3), pp. 211-217. https://doi.org/10.1016/j.ausmj.2012.02.001

Clerwall, C. (2014) "Enter the Robot Journalist: Users' Perceptions of automated content", Journalism Practice, 8(5), pp. 519-531. https://doi.org/10.1080/17512786.2014.883116

Daft, R. L., Lengel, R. H. (1983) "Information Richness: A New Approach to Managerial Behavior and Organization Design", College of Business Administration, Texas A\&M University, College Station, Texas, USA, Rep. TR-ONR-DG-02.

Daft, R. L., Lengel, R. H. (1986) "Organizational Information Requirements, Media Richness and Structural Design", Management Science, 32(5), pp. 554-571. https://doi.org/10.1287/mnsc.32.5.554

Doong, H-S., Wang, H-C., Foxall, G. R. (2010) "Psychological traits and loyalty intentions towards e-Government services", International Journal of Information Management, 30(5), pp. 457-464. https://doi.org/10.1016/j.ijinfomgt.2010.01.007

Edwards, A., Edwards, C., Westerman, D., Spence, P. R. (2019) "Initial Expectations, interactions, and beyond with social robots", Computers in Human Behavior, 90, pp. 308-314. https://doi.org/10.1016/j.chb.2018.08.042
In this light, therefore, it is important to understand people's perceptions and reactions to these kinds of services. Although we do not know what the realities of this world will be, having early indications of what voice versus other input modalities in the contemporary world is one of the best avenues of gaining insight into, and preparing for, this agent-driven world.

Edwards, C., Edwards, A., Spence, P. R., Westerman, D. (2016) "Initial Interaction Expectations with Robots: Testing the Human-toHuman Interaction Script", Communication Studies, 67(2), pp. 227-238.

https://doi.org/10.1080/10510974.2015.1121899

Fox, J., Ahn, S. J., Janssen, J. H., Yeykelis, L., Segovia, K. Y., Bailenson, J. N. (2015) "Avatars Versus Agents: A Meta-Analysis Quantifying the Effect of Agency on Social Influence", HumanComputer Interaction, 30(5), pp. 401-432. https://doi.org/10.1080/07370024.2014.921494

Gimpel, H., Huber, J., Sarikaya, S. (2016) "Customer Satisfaction in Digital Service Encounters: The Role of Media Richness, Social Presence, and Cultural Distance", In: The 24 ${ }^{\text {th }}$ European Conference on Information Sciences (ECIS), Istanbul, Turkey, Paper number: 91.

Gunkel, D. J. (2012) "Communication and Artificial Intelligence: Opportunities and Challenges for the 21st Century", communication $+1,1(1)$, pp. 1-25. https://doi.org/10.7275/R5QJ7F7R

Guzman, A. L. (2018) "Beyond Extraordinary: Theorizing Artificial Intelligence and the Self in Daily Life", In: Papacharissi, Z. (ed.) A Networked Self and Human Augmentics, Artificial Intelligence, Sentience, Routledge, New York, USA, pp. 83-96. https://doi.org/10.4324/9781315202082-7

Guzman, A. L., Lewis, S. C. (2020) "Artificial intelligence and communication: A Human-Machine Communication research agenda", New Media \& Society, 22(1), pp. 70-86. https://doi.org/10.1177/1461444819858691

Halpern, D., Katz, J. E. (2013) "Close But Not Stuck: Understanding Social Distance in Human-Robot Interaction Through a Computer Mediation Approach", Intervalla, 1, pp. 17-34.

Hunt, D., Atkin, D., Krishnan, A. (2012) "The Influence of ComputerMediated Communication Apprehension on Motives for Facebook Use", Journal of Broadcasting \& Electronic Media, 56(2), pp. 187-202. https://doi.org/10.1080/08838151.2012.678717

Hurt, H. T., Joseph, K., Cook, C. D. (1977) "Scales for the Measurement of Innovativeness", Human Communication Research, 4(1), pp. 58-65. https://doi.org/10.1111/j.1468-2958.1977.tb00597.x

Kalyanaraman, S. S., Wojdynski, B. W. (2015) "Affording Control: How Customization, Interactivity, and Navigability Affect Psychological Responses to Technology", The Handbook of the Psychology of Communication Technology, pp. 425-444. https://doi.org/10.1002/9781118426456.CH19 
Katz, J. E., Aspden, P., Reich, W. A. (1997) "Public attitudes toward voice-based electronic messaging technologies in the United States: A national survey of opinions about voice response units and telephone answering machines", Behaviour \& Information Technology, 16(3), pp. 125-144. https://doi.org/10.1080/014492997119860

McCroskey, J. C. (2005) "An Introduction to Rhetorical Communication", Routledge, New York, NY, USA. https://doi.org/10.4324/9781315663791

Miller, D. I., Talbot, V., Gagnon, M., Messier, C. (2013) "Administration of neuropsychological tests using interactive voice response technology in the elderly: validation and limitations", Frontiers in Neurology, 4, Article number: 107. https://doi.org/10.3389/fneur.2013.00107

Orr, C., Allen, D., Poindexter, S. (2001) "The Effect of Individual Differences on Computer Attitudes: An Empirical Study", Journal of Organizational and End User Computing (JOEUC), 13(2), pp. 26-39. https://doi.org/10.4018/joeuc.2001040103

Pieterson, W., Teerling, M., Ebbers, W. (2008) "Channel Perceptions and Usage: Beyond Media Richness Factors", In: Wimmer, M. A., Scholl, H. J., Ferro, E. (eds.) Electronic Government, Springer, Heidelberg, Berlin, Germany pp. 219-230. https://doi.org/10.1007/978-3-540-85204-9_19

Rice, R. E. (1992) "Task Analyzability, Use of New Media, and Effectiveness: A Multi-Site Exploration of Media Richness", Organization Science, 3(4), pp. 475-500. https://doi.org/10.1287/orsc.3.4.475

Rosen, L. D., Sears, D. C., Weil, M. M. (1993) "Treating technophobia: A longitudinal evaluation of the computerphobia reduction program", Computers in Human Behavior, 9(1), pp. 27-50. https://doi.org/10.1016/0747-5632(93)90019-O

Rogers, E. M. (2003) "Diffusion of Innovations", Free Press, New York, NY, USA.

Spence, P. R., Westerman, D., Edwards, C., Edwards, A. (2014) "Welcoming Our Robot Overlords: Initial Expectations About Interaction With a Robot", Communication Research Reports, 31(3), pp. 272-280. https://doi.org/10.1080/08824096.2014.924337

Spence, P. R. (2019) "Searching for questions, original thoughts, or advancing theory: Human-machine communication", Computers in Human Behavior, 90, pp. 285-287. https://doi.org/10.1016/j.chb.2018.09.014

STARTEK (2017) "Will Chatbots Replace Humans Any Time Soon? Highlights from the STARTEK U.S. customer engagement survey", [online] Available at: https://www.startek.com/lp/ customer-engagement-survey-2017 [Accessed: 20 July 2020]

Sundar, S. S. (2007) "The MAIN Model: A Heuristic Approach to Understanding Technology Effects on Credibility", In: Metzger, M. J., Flanagin, A. J. (eds.) Digital Media, Youth, and Credibility, The John D. and Catherine T. MacArthur Foundation Series on Digital Media and Learning, The MIT Press, Cambridge, MA, USA, pp. 73-100.

https:/doi.org/10.1162/dmal.9780262562324.073
Sundar, S. S., Kim, J. (2019) "Machine Heuristic: When We Trust Computers More than Humans with Our Personal Information", In: Proceedings of the 2019 CHI Conference on Human Factors in Computing Systems, Glasgow, Scotland, UK, pp. 1-9. https://doi.org/10.1145/3290605.3300768

Sundar, S. S., Nass, C. (2001) "Conceptualizing Sources in Online News", Journal of Communication, 51(1), pp. 52-72. https://doi.org/10.1111/j.1460-2466.2001.tb02872.x

Van Braak, J. (2001) "Individual Characteristics Influencing Teachers' Class Use of Computers", Journal of Educational Computing Research, 25(2), pp. 141-157. https://doi.org/10.2190/81YV-CGMU-5HPM-04EG

Vickery, S. K., Droge, C., Stank, T. P., Goldsby, T. J., Markland, R. E. (2004) "The Performance Implications of Media Richness in a Business-to-Business Service Environment: Direct Versus Indirect Effects", Management Science, 50(8), pp. 1106-1119. https://doi.org/10.1287/mnsc.1040.0248

Waddell, T. F. (2018) "A Robot Wrote This? How perceived machine authorship affects news credibility", Digital Journalism, 6(2), pp. $236-255$. https://doi.org/10.1080/21670811.2017.1384319

Waddell, T. F. (2019) "Can an Algorithm Reduce the Perceived Bias of News? Testing the Effect of Machine Attribution on News Readers' Evaluations of Bias, Anthropomorphism, and Credibility", Journalism \& Mass Communication Quarterly, 96(1), pp. 82-100. https://doi.org/10.1177/1077699018815891

Walsh, J., Andersen, B. L., Katz, J. E., Groshek, J. (2018) "Personal Power and Agency When Dealing with Interactive Voice Response Systems and Alternative Modalities", Media and Communication, 6(3), pp. 60-68.

https://doi.org/10.17645/mac.v6i3.1205

Walther, J. B. (1992) "Interpersonal Effects in Computer-Mediated Interaction: A Relational Perspective", Communication Research, 19(1), pp. 52-90. https://doi.org/10.1177/009365092019001003

Walther, J. B. (1994) "Anticipated Ongoing Interaction Versus Channel Effects on Relational Communication in Computer-Mediated Interaction", Human Communication Research, 20(4), pp. 473-501. https://doi.org/10.1111/j.1468-2958.1994.tb00332.x

Yi, M. Y., Fiedler, K. D., Park, J. S. (2006) "Understanding the Role of Individual Innovativeness in the Acceptance of IT-Based Innovations: Comparative Analyses of Models and Measures", Decision Sciences, 37(3), pp. 393-426. https://doi.org/10.1111/j.1540-5414.2006.00132.x 